\title{
On Games and Equilibria with Coherent Lower Expectations
}

\author{
Giuseppe De Marco ${ }^{1,2}$ and Maria Romaniello ${ }^{3}$ \\ ${ }^{1}$ Department of Management and Quantitative Studies, Parthenope University of Naples, Via Generale Parisi 13, 80132 Naples, Italy \\ ${ }^{2}$ CSEF, University of Naples Federico II, Via Cintia, 80126 Naples, Italy \\ ${ }^{3}$ Department of Economics, Second University of Naples, Corso Gran Priorato di Malta, 81043 Capua, Italy
}

Correspondence should be addressed to Giuseppe De Marco; demarco@uniparthenope.it

Received 31 March 2015; Accepted 16 June 2015

Academic Editor: Eva Onaindia

Copyright (C) 2015 G. De Marco and M. Romaniello. This is an open access article distributed under the Creative Commons Attribution License, which permits unrestricted use, distribution, and reproduction in any medium, provided the original work is properly cited.

\begin{abstract}
Different solution concepts for strategic form games have been introduced in order to weaken the consistency assumption that players' beliefs, about their opponents strategic choices, are correct in equilibrium. The literature has shown that ambiguous beliefs are an appropriate device to deal with this task. In this note, we introduce an equilibrium concept in which players do not know the opponents' strategies in their entirety but only the coherent lower expectations of some random variables that depend on the actual strategies taken by the others. This equilibrium concept generalizes the already existing concept of equilibrium with partially specified probabilities by extending the set of feasible beliefs and allowing for comparative probability judgements. We study the issue of the existence of the equilibrium points in our framework and find sufficient conditions which involve the continuity of coherent lower expectations and a Slater-like condition for the systems of inequalities defining beliefs.
\end{abstract}

\section{Introduction}

The concept of Nash equilibrium relies on two fundamental ideas. Firstly, the agents choose their optimal strategies according to the beliefs they have about the strategic choice made by the other players. Secondly, beliefs are consistent; that is, agents have correct expectations about the actual strategies chosen by their opponents. One of the main criticisms to the Nash equilibrium concept has always been the strength of such consistency condition as, in many settings, it is not clear why players must have exactly correct beliefs about each other. Therefore, different solution concepts have been introduced in order to weaken such consistency condition by taking into account perturbed beliefs. In a random belief equilibrium, [1], players' beliefs about others' strategy choices are randomly drawn from a belief distribution that is dispersed around a central strategy profile called the focus. From a different perspective, other solutions concepts are based on the assumption that players have ambiguous beliefs about opponents behavior. A strand of this literature follows the Choquet expected utility approach [2-5]; that is, beliefs are represented by capacities (nonadditive measures) and expectations are expressed in terms of Choquet integrals. In the maxmin expected utility approach [6-9] beliefs are described by sets of probability distributions, so that ambiguity averse agents evaluate these sets by the worst feasible expectations. The approach proposed by Lehrer [9] is based on the partial specification of players' actions; that is, players' beliefs about the strategic choice of their opponents are given by partially specified probabilities. More precisely, players do not know the mixed strategy profile chosen by their opponents in their entirety but only the expectations of some (specified) random variables that depend on the actual choice taken by the others. This is the case, for instance, of players who are able to assess the probability of some subsets of pure strategies but do not know in which way these probabilities are distributed within those subsets.

We point out in this paper that partially specified probabilities are a particular case of coherent lower expectations which, in turn, emerge as a key concept in the literature on imprecise probabilities; as a consequence, equilibria with partially specified probabilities can be immediately generalized 
to a concept of equilibrium in which each agent knows the coherent lower expectations, instead of the expectations, of the random variables which are specified. This is done in the present paper by introducing the so-called concept of equilibrium with coherent lower expectations. At first sight, this concept is similar to Lehrer's one as ambiguity stems from the actual strategies played but, at the same time, it differs from Lehrer's concept as the true strategy profile is not necessarily contained in the belief of every player. Most importantly, it is more flexible than Lehrer's one since lower expectations can model comparative probability judgements, such as "a given event is at least as probable as ...," which cannot always be reconducted to partially specified probabilities. This is the case, for instance, of players who are able to assess that the probability of some subsets of pure strategies is at least as probable as a given value which might depend on the strategy profile.

Although the features of our concept of equilibrium are rather general, this is not reflected in very restrictive assumptions for the existence of the corresponding equilibrium points. This is our main result: equilibria with coherent lower expectations exist, provided that coherent lower expectations are continuous and a Slater-like condition for the beliefs is satisfied: the system of linear inequalities which defines the beliefs of each player has interior points belonging to the simplex. More precisely, we firstly point out that an existence result is obtained rather easily once it is shown that equilibria with coherent lower expectations have an equivalent formulation as equilibria of games under ambiguous belief correspondences [10]; in particular, we apply the existence result for this concept as presented in De Marco and Romaniello [11] (Theorem 6). However, this approach does not allow having explicit sufficient conditions on the founding concepts of the particular model studied in this paper, which are the coherent lower expectation functions and the set of specified random variables. Our main result, which is presented in Proposition 7, is precisely to find conditions on such founding concepts in order that the corresponding game under ambiguous belief correspondences satisfies the existence theorem in De Marco and Romaniello [11]. A final example shows that Proposition 7 is useful also for applications since it turns out that it is much more simple to check whether the sufficient conditions in Proposition 7 are satisfied than looking at the assumptions of the general existence theorem in De Marco and Romaniello [11].

As a final remark, the paper by Groes et al. [8] proposes an approach similar to ours and that of Lehrer [9] since expectation about opponents' strategic choices is described by lower probabilities. However, Walley [12] points out at page 134 that "coherent lower expectations are more general and more informative than coherent lower probabilities,"1 so that our concept generalizes also the one in Groes et al. [8].

The paper is organized as follows. In Section 2, we define the model and the equilibrium concept; then, we relate it to the equilibrium concepts in De Marco and Romaniello [10] and Lehrer [9]. Section 3 is devoted to the equilibrium existence theorem.

\section{Ambiguous Beliefs and Equilibria}

2.1. The Equilibrium Concept. We consider a finite set of players $I=\{1, \ldots, n\}$; for every player $i, \Psi_{i}=\left\{\psi_{i}^{1}, \ldots, \psi_{i}^{k_{i}}\right\}$ is the (finite) pure strategy set of player $i, \Psi=\prod_{i \in I} \Psi_{i}$ and $\Psi_{-i}=$ $\prod_{j \neq i} \Psi_{j}$. Denote with $X_{i}$ the set of mixed strategies of player $i$, where each strategy $x_{i} \in X_{i}$ is a vector $x_{i}=\left(x_{i}\left(\psi_{i}\right)\right)_{\psi_{i} \in \Psi_{i}} \in \mathbb{R}_{+}^{k_{i}}$ such that $\sum_{\psi_{i} \in \Psi_{i}} x_{i}\left(\psi_{i}\right)=1$. Denote with $X=\prod_{j=1}^{n} X_{j}$ and with $X_{-i}=\prod_{j \neq i} X_{j}$, then each mixed strategy profile $x \in X$ can also be denoted by $x=\left(x_{i}, x_{-i}\right)$, where $x_{i} \in X_{i}$ and $x_{-i} \in$ $X_{-i}$. Finally, we assume that each player $i$ is endowed with a payoff function $f_{i}: \Psi \rightarrow \mathbb{R}$.

Agents have ambiguous expectations about the strategy choice of their opponents. In particular, the information available to player $i$ about player $j$ 's strategic choice is given by the coherent lower expectations (see Walley [12] and references therein) of specified random variables over $\Psi_{j}$. We denote with $\mathcal{Y}_{i j}$ the set of the random variables over $\Psi_{j}$ which are specified to player $i$. More precisely, the coherent lower expectations of player $i$ about player $j$ 's strategic choice are assigned by a function $P_{i j}: \mathscr{Y}_{i j} \times X_{j} \rightarrow \mathbb{R}$ provided that

$$
P_{i j}\left(Y_{i j}, x_{j}\right)=\min _{y_{j} \in K_{i j}\left(x_{j}\right)} E_{y_{j}}\left[Y_{i j}\right]
$$

where $E_{y_{j}}\left[Y_{i j}\right]$ is the expectation of $Y_{i j}$ under $y_{j}$, that is, $E_{y_{j}}\left[Y_{i j}\right]=\sum_{d=1}^{k_{j}} y_{j}\left(\psi_{j}^{d}\right) Y_{i j}\left(\psi_{j}^{d}\right)$, and the set-valued map $K_{i j}$ : $X_{j} \leadsto X_{j}$ is defined by

$$
\begin{array}{r}
K_{i j}\left(x_{j}\right) \\
=\left\{y_{j} \in X_{j} \mid E_{y_{j}}\left[Y_{i j}\right] \geqslant P_{i j}\left(Y_{i j}, x_{j}\right) \quad \forall Y_{i j} \in \mathscr{Y}_{i j}\right\} \\
\forall x_{j} \in X_{j} .
\end{array}
$$

In this case, given a mixed strategy $x_{j}$ of player $j$, player $i$ does not know $x_{j}$ in its entirety but knows the coherent lower expectations $P_{i j}\left(Y_{i j}, x_{j}\right)$ of each $Y_{i j} \in \mathscr{Y}_{i j}$ and the ambiguous belief of player $i$ about player $j$ 's strategy choice is the set $K_{i j}\left(x_{j}\right)$ of all player $j$ 's mixed strategies that are consistent with the coherent lower probability $P_{i j}\left(Y_{i j}, x_{j}\right)$.

Then, the utility function of player $i$ is defined by

$$
U_{i}(x)=\min _{\left(y_{j}\right)_{j \neq i} \in \prod_{j \neq i} K_{i j}\left(x_{j}\right)} x_{i}\left(\psi_{i}\right)\left[\prod_{j \neq i} y_{j}\left(\psi_{j}\right)\right] f(\psi)
$$

$\forall x \in X$

So, we can consider the following strategic form game:

$$
\Gamma=\left\{I ;\left(X_{i}\right)_{i \in I},\left(U_{i}\right)_{i \in I}\right\} .
$$

Hence, we introduce the following.

Definition 1. Let $\mathscr{Y}_{i j}$ be the set of random variables defined over $\Psi_{j}$ whose coherent lower expectations are specified to player $i$, for every pair $(i, j)$. Then, any Nash equilibrium 
$x \in X$ of the corresponding game $\Gamma$ (defined by (4)) is said to be an equilibrium with coherent lower expectations with respect to the sets $\left(\mathscr{Y}_{i j}\right)_{i, j}$; that is, $x \in X$ is an equilibrium with coherent lower expectations if, for every player $i$,

$$
U_{i}\left(x_{i}, x_{-i}\right) \geqslant U_{i}\left(x_{i}^{\prime}, x_{-i}\right) \quad \forall x_{i}^{\prime} \in X_{i}
$$

2.2. The Equivalent Formulation under Ambiguous Belief Correspondences. Now we show that equilibria with coherent lower expectations have an equivalent formulation as equilibria in games under ambiguous beliefs correspondences as firstly introduced in De Marco and Romaniello $[10]^{2}$. This formulation is key because it allows applying directly the existence result in De Marco and Romaniello [11] to obtain an existence result for equilibria with coherent lower expectations ${ }^{3}$.

Denote with $\Omega=\left\{\left(f_{1}(\psi), \ldots, f_{n}(\psi)\right) \mid \psi \in \Psi\right\}$ the set of all the outcomes of the game and denote with $\mathscr{P}$ the set of all the probability distributions over $\Omega$. Define the ambiguous belief correspondence over outcomes $\mathscr{B}_{i}: X \rightsquigarrow \mathscr{P}$ as follows:

$$
\begin{aligned}
& \varrho \in \mathscr{B}_{i}(x) \Longleftrightarrow \exists\left(y_{j}\right)_{j \neq i} \in \prod_{j \neq i} K_{i j}\left(x_{j}\right) \\
& \text { s.t. } \varrho(\psi)=x_{i}\left(\psi_{i}\right)\left[\prod_{j \neq i} y_{j}\left(\psi_{j}\right)\right] \quad \forall \psi \in \Psi .
\end{aligned}
$$
by

Assume that each player $i$ has payoff $F_{i}: X \rightarrow \mathbb{R}$ defined

$$
F_{i}(x)=\min _{\varrho \in \mathscr{B}_{i}(x)} E_{i}(\varrho) \quad \forall x \in X
$$

where $E_{i}[\varrho]=\sum_{\psi \in \Psi} \varrho(\psi) f_{i}(\psi)$; then we consider the following game under ambiguous beliefs correspondences $\mathscr{B}=$ $\left(\mathscr{B}_{1}, \ldots, \mathscr{B}_{n}\right)$ and pessimistic players:

$$
\Gamma^{\mathscr{B}}=\left\{I ;\left(X_{i}\right)_{i \in I} ;\left(F_{i}\right)_{i \in I}\right\} .
$$

This game is a classical strategic form game and we call equilibria under ambiguous belief correspondences $\mathscr{B}_{i}$ the classical Nash equilibria of $\Gamma^{\mathscr{B}}$. That is, $\left(x_{i}, x_{-i}\right)$ is an equilibrium under ambiguous belief correspondences $\mathscr{B}_{i}$ if, for every player $i$,

$$
F_{i}\left(x_{i}, x_{-i}\right) \geqslant F_{i}\left(x_{i}^{\prime}, x_{-i}\right) \quad \forall x_{i}^{\prime} \in X_{i} .
$$

It immediately follows from the definition that a strategy profile $x$ is an equilibrium with coherent lower expectations if and only if it is an equilibrium under ambiguous belief correspondences defined by (6).

2.3. Equilibria with Partially Specified Probabilities. This subsection highlights the relation of our equilibrium notion with respect to Lehrer's concept of equilibrium with partially specified probabilities. Let $\mathcal{Y}_{i j}^{L}$ be the set of random variables defined over $\Psi_{j}$ whose expectations are specified to player $i$; that is, given a mixed strategy $x_{j}$, player $i$ does not know $x_{j}$ in its entirety but knows the expectation of each $Y_{i j} \in \mathcal{Y}_{i j}^{L}$. Then, the ambiguous belief $K_{i j}^{L}\left(x_{j}\right)$ of player $i$ about player $j$ 's strategy choice is the set of all player $j$ 's mixed strategies that are consistent with $x_{j}$ and $\mathscr{Y}_{i j}^{L}$ in the following way:

$$
\begin{aligned}
& K_{i j}^{L}\left(x_{j}\right) \\
& \quad=\left\{y_{j} \in X_{j} \mid E_{y_{j}}\left[Y_{i j}\right]=E_{x_{j}}\left[Y_{i j}\right] \forall Y_{i j} \in \mathcal{Y}_{i j}^{L}\right\},
\end{aligned}
$$

where $E_{y_{j}}\left[Y_{i j}\right]$ and $E_{x_{j}}\left[Y_{i j}\right]$ represent the expectation of $Y_{i j}$ with respect to $y_{j}$ and $x_{j}$, respectively. Then, the utility function of player $i$ is constructed as

$$
\begin{aligned}
U_{i}^{L}(x)=\min _{\left(y_{j}\right)_{j \neq i} \in \prod_{j \neq i} K_{i j}^{L}\left(x_{j}\right)} x_{i}\left(\psi_{i}\right)\left[\prod_{j \neq i} y_{j}\left(\psi_{j}\right)\right] & f(\psi) \\
& \forall x \in X
\end{aligned}
$$

and the game is

$$
\Gamma^{L}=\left\{I ;\left(X_{i}\right)_{i \in I},\left(U_{i}^{L}\right)_{i \in I}\right\} .
$$

Definition 2 (see Lehrer [9]). Let $\mathscr{Y}_{i j}$ be the set of random variables defined over $\Psi_{j}$ whose expectations are specified to player $i$, for every pair $(i, j)$. Then, any Nash equilibrium $x \in X$ of the corresponding game $\Gamma^{L}$ (defined by (12)) is said to be an equilibrium with partially specified probabilities with respect to the sets $\left(\mathscr{Y}_{i j}\right)_{i, j}$.

It is clear that equilibria with partially specified probabilities are a special case of equilibria with coherent lower expectations. More precisely, let $x$ be an equilibrium with partially specified probabilities with respect to the sets $\mathscr{Y}_{i j}^{L}$ for every pair $(i, j)$ and let $K_{i j}^{L}\left(x_{j}\right)$ be the corresponding set of beliefs as defined in (10). Now, let $\mathscr{Y}_{i j}$ be the set of random variables defined as follows:

$$
\begin{aligned}
Y_{i j} \in \mathscr{Y}_{i j} & \Longleftrightarrow Y_{i j} \in \mathscr{Y}_{i j}^{L} \\
\text { or }-Y_{i j} \in \mathcal{Y}_{i j}^{L} ; &
\end{aligned}
$$

then, for every $x_{j} \in X_{j}$, let the coherent lower probabilities $P_{i j}\left(\cdot, x_{j}\right): \mathscr{Y}_{i j} \rightarrow \mathbb{R}$ be defined by $P_{i j}\left(Y_{i j}, x_{j}\right)=E_{x_{j}}\left[Y_{i j}\right]$ for all $Y_{i j} \in \mathscr{Y}_{i j}$. It follows that the corresponding set of beliefs $K_{i j}\left(x_{j}\right)$ defined by (2) coincides with $K_{i j}^{L}\left(x_{j}\right)$, so that equilibria with partially specified equilibria are equilibria with coherent lower expectations.

Remark 3. We have shown that partially specified probabilities can be reconducted to coherent lower expectations. Conversely, lower expectations can model comparative probability judgements which cannot always be described by partially specified probabilities; as already noticed in the Introduction, this is the case, for instance, of judgements like "... a given event is at least as probable as ...." The advantages and drawbacks of coherent lower expectations are studied extensively in the literature (see, e.g., Walley [12] and 
references therein); here we just point out that, in our case, coherent lower expectations can model situations in which players are able to assess that the probability of some subsets of pure strategies is at least as probable as a given value which might depend on the strategy profile. Consider the following simple example: suppose that an agent, say $i$, can only perceive the minimum probability under which his opponent, say $j$, will play each pure strategy. This means that player $i$ has the following beliefs about player $j$ 's strategic choice:

$$
\begin{aligned}
& K_{i j}\left(x_{j}\right)=\left\{y_{j} \in X_{j} \mid y_{j}\left(\psi_{j}^{d}\right) \geqslant \min _{d \in\left\{1, \ldots, k_{j}\right\}} x_{j}\left(\psi_{j}^{d}\right) \forall d\right. \\
& \left.\quad \in\left\{1, \ldots, k_{j}\right\}\right\} \quad \forall x_{j} \in X_{j} .
\end{aligned}
$$

Now, the previous belief $K_{i j}\left(x_{j}\right)$ comes out from coherent lower expectations by setting, in formula (2),

$$
\mathscr{Y}_{i j}=\left\{\chi\left(\psi_{j}^{d}\right) \mid d=1, \ldots, k_{j}\right\}
$$

where each $\chi\left(\psi_{j}^{d}\right)$ is the characteristic function of the event $\left\{\psi_{j}^{d}\right\}$ and

$$
P_{i j}\left(\chi\left(\psi_{j}^{d}\right), x_{j}\right)=\min _{d \in\left\{1, \ldots, k_{j}\right\}} x_{j}\left(\psi_{j}^{d}\right)
$$

\section{Equilibrium Existence}

3.1. Preliminaries on Set-Valued Maps. We start by recalling well-known definitions and results on set-valued maps which we use below. Following Aubin and Frankowska [13] ${ }^{4}$, recall that if $Z$ and $Y$ are two metric spaces and $\mathscr{C}: Z \backsim Y$ is a set-valued map, then

(i) $\operatorname{Liminf}_{z \rightarrow z^{\prime}} \mathscr{C}(z)=\left\{y \in Y \mid \lim _{z \rightarrow z^{\prime}} d(y, \mathscr{C}(z))=0\right\}$,

(ii) $\operatorname{Lim}_{z \rightarrow z^{\prime}} \sup \mathscr{C}(z)$

$$
=\left\{y \in Y \mid \operatorname{Liminf}_{z \rightarrow z^{\prime}} d(y, \mathscr{C}(z))=0\right\}
$$

and $\operatorname{Liminf} \operatorname{siz}_{z \rightarrow z^{\prime}} \mathscr{C}(z) \subseteq \mathscr{C}\left(z^{\prime}\right) \subseteq \operatorname{Lim} \sup _{z \rightarrow z^{\prime}} \mathscr{C}(z)$. Moreover, we introduce the following.

Definition 4. Given the set-valued map $\mathscr{C}: Z \backsim Y$, then

(i) $\mathscr{C}$ is lower semicontinuous in $z^{\prime}$ if $\mathscr{C}\left(z^{\prime}\right) \subseteq$ Liminf $z \rightarrow z^{\prime} \mathscr{C}(z)$; that is, $\mathscr{C}$ is lower semicontinuous in $z^{\prime}$ if for every $y \in \mathscr{C}\left(z^{\prime}\right)$ and every sequence $\left(z_{v}\right)_{v \in \mathbb{N}}$ converging to $z^{\prime}$ there exists a sequence $\left(y_{v}\right)_{v \in \mathbb{N}}$ converging to $y$ such that $y_{v} \in \mathscr{C}\left(z_{v}\right)$ for every $v \in \mathbb{N}$; moreover, $\mathscr{C}$ is lower semicontinuous in $Z$ if it is lower semicontinuous for all $z^{\prime}$ in $Z$;

(ii) $\mathscr{C}$ is closed in $z^{\prime}$ if $\operatorname{Lim} \sup _{z \rightarrow z^{\prime}} \mathscr{C}(z) \subseteq \mathscr{C}\left(z^{\prime}\right)$; that is, $\mathscr{C}$ is closed in $z^{\prime}$ if, for every sequence $\left(z_{\nu}\right)_{v \in \mathbb{N}}$ converging to $z^{\prime}$ and every sequence $\left(y_{v}\right)_{v \in \mathbb{N}}$ converging to $y$ such that $y_{v} \in \mathscr{C}\left(z_{v}\right)$ for every $v \in \mathbb{N}$, it follows that $y \in \mathscr{C}\left(z^{\prime}\right)$; moreover, $\mathscr{C}$ is closed in $Z$ if it is closed for all $z^{\prime}$ in $Z$;

(iii) $\mathscr{C}$ is upper semicontinuous in $z^{\prime}$ if for every open set $U$ such that $\mathscr{C}\left(z^{\prime}\right) \subseteq U$ there exists $\eta>0$ such that $\mathscr{C}(z) \subseteq U$ for all $z \in B_{Z}\left(z^{\prime}, \eta\right)=\left\{\zeta \in Z \mid\left\|\zeta-z^{\prime}\right\|<\eta\right\} ;$

(iv) $\mathscr{C}$ is continuous (in the sense of Painlevé-Kuratowski) in $z^{\prime}$ if it is lower semicontinuous and upper semicontinuous in $z^{\prime}$.

Finally, recall the following result: if $Z$ is closed, $Y$ is compact and the set-valued map $\mathscr{C}: Z \backsim Y$ has closed values; then, $\mathscr{C}$ is upper semicontinuous in $z \in Z$ if and only if $\mathscr{C}$ is closed in $z^{5}$.

The next definition will also be used.

Definition 5. Let $Z$ be a convex set; then the set-valued map $\mathscr{C}: Z \backsim Y$ is said to be concave if

$$
t \mathscr{C}(\bar{z})+(1-t) \mathscr{C}(\widehat{z}) \subseteq \mathscr{C}(t \bar{z}+(1-t) \widehat{z})
$$

$$
\forall \bar{z}, \widehat{z} \in Z, \forall t \in[0,1],
$$

while it is convex ${ }^{6}$ if

$$
\begin{aligned}
& \mathscr{C}(t \bar{z}+(1-t) \widehat{z}) \subseteq t \mathscr{C}(\bar{z})+(1-t) \mathscr{C}(\widehat{z}) \\
& \forall \bar{z}, \widehat{z} \in Z, \forall t \in[0,1] .
\end{aligned}
$$

3.2. The Existence Theorem. As a direct application of Theorem 3.6 in De Marco and Romaniello [11] we have the following.

Theorem 6. Assume that, for every player $i$,

(i) $\mathscr{B}_{i}$ is a continuous set-valued map with not empty and closed images for every $x \in X$;

(ii) $\mathscr{B}_{i}\left(\cdot, x_{-i}\right)$ is a convex set-valued map in $X_{i}$ for every $x_{-i} \in X_{-i}$.

Then, the game $\Gamma^{\mathscr{B}}$ as defined in (4) has at least an equilibrium.

Proof. The proof follows directly by applying Theorem 3.6 in De Marco and Romaniello [11], since the utility function in (7) is a particular case of variational preferences in which the index of ambiguity aversion is identically equal to 0 .

The previous theorem does not allow understanding which are the explicit conditions that must be imposed on the grounding concepts of the model (coherent lower probabilities and specified random variables) in order to have the existence of equilibria. The next proposition is the main contribution of this paper as it gives explicit sufficient conditions on the coherent lower probabilities $P_{i j}$ and on the specified random variables in $\mathscr{Y}_{i j}$ which guarantee that the assumptions of the previous theorem hold, so that equilibria with coherent lower expectations exist. Moreover, Example 8 will show that the next proposition is useful also in the applications as it makes it more simple to check whether equilibria exist. 
Proposition 7. Let $K_{i j}$ be defined by (2), for every $j \neq i$, and let the corresponding $\mathscr{B}_{i}$ be defined by (6); then the following results hold.

(1) Assume that, for every player $j \neq i$,

(i) the coherent lower probability function $P_{i j}\left(Y_{i j}, \cdot\right)$ is continuous in the point $x_{j} \in X_{j}$ for every $Y_{i j} \in$ $y_{i j}$,

(ii) there exists $y_{j} \in K_{i j}\left(x_{j}\right)$ such that

$$
\begin{gathered}
y_{j}\left(\psi_{j}^{d}\right)>0 \quad \forall d \in\left\{1, \ldots, k_{j}\right\}, \\
E_{y_{j}}\left[Y_{i j}\right]>P\left(Y_{i j}, x_{j}\right) \quad \forall Y_{i j} \in \mathscr{Y}_{i j} .
\end{gathered}
$$

Then, the belief correspondence $\mathscr{B}_{i}$ is a continuous setvalued map with a not empty and closed image in the point $x=\left(x_{1}, \ldots, x_{n}\right) \in X$. Hence, if (i) and (ii) hold for every $j \neq i$ and for every $x_{j} \in X_{j}$, then $\mathscr{B}_{i}$ is a continuous set-valued map with not empty and closed images for every $x \in X$.

(2) $\mathscr{B}_{i}\left(\cdot, x_{-i}\right)$ is a convex set-valued map in $X_{i}$, for every $x_{-i} \in X_{-i}$.

Proof. First of all recall that

$$
\begin{aligned}
& \varrho \in \mathscr{B}_{i}(x) \Longleftrightarrow \exists\left(y_{j}\right)_{j \neq i} \in \prod_{j \neq i} K_{i j}\left(x_{j}\right) \\
& \text { s.t. } \varrho(\psi)=x_{i}\left(\psi_{i}\right)\left[\prod_{j \neq i} y_{j}\left(\psi_{j}\right)\right] \quad \forall \psi \in \Psi .
\end{aligned}
$$

The assumptions imply that $K_{i j}\left(x_{j}\right) \neq \emptyset$ so $\mathscr{B}_{i}(x) \neq \emptyset$ for every $x \in X$.

Step $1\left(\mathscr{B}_{i}\right.$ is lower semicontinuous). Let $\left|\mathscr{Y}_{i j}\right|=\gamma_{j}$ be the cardinality of $\mathscr{Y}_{i j}$; it follows that $K_{i j}\left(x_{j}\right)$ is completely defined by the following system of $k_{j}+\gamma_{j}$ linear inequalities in the $k_{j}$ unknowns $y_{j}=\left(y_{j}\left(\psi_{j}^{1}\right), \ldots, y_{j}\left(\psi_{j}^{k_{j}}\right)\right)$ :

$$
\begin{gathered}
y_{j}\left(\psi_{j}^{d}\right) \geqslant 0 \quad \forall d \in\left\{1, \ldots, k_{j}\right\} \\
E_{y_{j}}\left[Y_{i j}\right] \geqslant P\left(Y_{i j}, x_{j}\right) \quad \forall Y_{i j} \in \mathscr{Y}_{i j}
\end{gathered}
$$

and by the linear equality

$$
y_{j}\left(\psi_{j}^{1}\right)+\cdots+y_{j}\left(\psi_{j}^{k_{j}}\right)=1
$$

It follows immediately that $K_{i j}\left(x_{j}\right)$ is a closed set and therefore compact set.

Now, denote with $C$ the $\left(k_{j}+\gamma_{j}\right) \times k_{j}$ matrix of the coefficients in system (22) and with $\mathbf{0}_{k_{j}}$ the row null vector of dimension $k_{j}$. Let $b\left(x_{j}\right)$ be the $\left(k_{j}+\gamma_{j}\right)$-dimensional row vector defined as follows:

$$
b\left(x_{j}\right)=\left(\mathbf{0}_{k_{j}},\left(P_{i j}\left(Y_{i j}, x_{j}\right)\right)_{Y_{i j} \in \mathscr{Y}_{i j}}\right) .
$$

If $y_{j}^{T}$ and $b^{T}\left(x_{j}\right)$ are the transpose of $y_{j}$ and $b\left(x_{j}\right)$, respectively, then system (22) can be denoted as follows: $C y_{j}^{T} \geqq b^{T}\left(x_{j}\right)$. Denote also with $\mathbf{1}_{k_{j}} y_{j}^{T}=1$ the linear equation (23). Theorem 2 in Robinson [14] tells that if a system of linear equalities and inequalities is such that the coefficient matrix of the equalities has full rank and there exists a feasible point satisfying all the strict inequalities, then the system is stable as defined at page 755 in Robinson [14] $]^{7}$. This is our case, because assumption (ii) guarantees that there exists a vector $\bar{y}_{j}$ such that

$$
\begin{aligned}
& C \bar{y}_{j}^{T}>b^{T}\left(x_{j}\right), \\
& \mathbf{1}_{k_{j}} \bar{y}_{j}^{T}=1,
\end{aligned}
$$

so the system is stable which, in our case, means that for every vector $y_{j}$ satisfying $C y_{j}^{T} \geqq b^{T}\left(x_{j}\right)$ and $\mathbf{1}_{k_{j}} y_{j}^{T}=1$ and every $\varepsilon>$ 0 there exists $\delta(\varepsilon)>0^{8}$ such that for every $\left(k_{j}+\gamma_{j}\right)$-dimensional row vector $\tilde{b}$ with $\left\|\widetilde{b}-b\left(x_{j}\right)\right\|<\delta(\varepsilon)$ there exists $\tilde{y}_{j}$ satisfying (a) $C \tilde{y}_{j}^{T} \geqq \widetilde{b}^{T}$ and $\mathbf{1}_{k_{j}} \tilde{y}_{j}^{T}=1$ and (b) $\left\|\tilde{y}_{j}-y_{j}\right\|<\varepsilon$.

So $K_{i j}$ is a lower semicontinuous set-valued map in $x_{j}$. In fact, let $\left(x_{j, v}\right)_{v \in \mathbb{N}}$ be a sequence converging to $x_{j}$ and $y_{j} \epsilon$ $K_{i j}\left(x_{j}\right)$. For every $v \in \mathbb{N}$, arg $\min _{z \in K_{i j}\left(x_{j, v}\right)}\left\|z-y_{j}\right\|$ is not empty since $K_{i j}\left(x_{j, v}\right)$ is not empty and compact. Let $\left(y_{j, v}\right)_{v \in \mathbb{N}}$ be a sequence such that $y_{j, v} \in \arg \min _{z \in K_{i j}\left(x_{j, v}\right)}\left\|z-y_{j}\right\|$ for every $\nu \in \mathbb{N}$; we show that $y_{j, v} \rightarrow y_{j}$. Since $P_{i j}\left(Y_{i, j}, \cdot\right)$ is continuous in $x_{j}$ for every $Y_{i j} \in \mathscr{Y}_{i j}$, then the vector valued function $b(\cdot)$ defined by (24) is continuous. So, given $\delta(\varepsilon)>0$, there exists $\bar{v}$ such that for every $v \geqslant \bar{v}$ it follows that $\left\|b\left(x_{j, v}\right)-b\left(x_{j}\right)\right\|<\delta(\varepsilon)$. Therefore, since Robinson's [14] stability holds, then for every $\varepsilon>0$ there exists $\bar{v}$ such that for every $\nu \geqslant \bar{v}$ there exists $\tilde{y}_{j, v} \in K_{i j}\left(x_{j, v}\right)$ satisfying $\left\|\tilde{y}_{j, v}-y_{j}\right\|<\varepsilon$. Having $\left\|y_{j, v}-y_{j}\right\| \leqslant$ $\left\|\tilde{y}_{j, v}-y_{j}\right\|<\varepsilon$, we get that for every $\varepsilon>0$ there exists $\bar{v}$ such that for every $v \geqslant \bar{v}$ it follows that $\left\|y_{j, v}-y_{j}\right\|<\varepsilon$. So $y_{j, v} \rightarrow y_{j}$ and $K_{i j}$ is lower semicontinuous in $x_{j}$. Moreover, if (i) and (ii) are satisfied for every $x_{j} \in X_{j}$, then $K_{i j}$ is lower semicontinuous in $X_{j}$.

Now, let $\varrho \in \mathscr{B}_{i}(x)$ be the probability distribution defined as in (21) by

$$
\varrho(\psi)=x_{i}\left(\psi_{i}\right)\left[\prod_{j \neq i} y_{j}\left(\psi_{j}\right)\right]
$$

$$
\forall \psi \in \Psi \text {, with } y_{j} \in K_{i j}\left(x_{j}\right) j \neq i \text {; }
$$

let $\left(x_{v}\right)_{v \in \mathbb{N}}$ be a sequence converging to $x$. Since for every $j \neq i, K_{i j}$ is lower semicontinuous in $x_{j}$, then there exists a sequence $\left(y_{j, v}\right)_{v \in \mathbb{N}}$ converging to $y_{j}$ with $y_{j, v} \in K_{i j}\left(x_{j, v}\right)$ for every $\nu \in \mathbb{N}$. Define $\varrho_{\nu}$ as follows:

$$
\varrho_{\nu}(\psi)=x_{i, \nu}\left(\psi_{i}\right)\left[\prod_{j \neq i} y_{j, \nu}\left(\psi_{j}\right)\right] \quad \forall \psi \in \Psi .
$$

It follows that $\varrho_{\nu} \rightarrow \varrho$ and $\varrho_{\nu} \in \mathscr{B}_{i}\left(x_{\nu}\right)$ for every $\nu \in \mathbb{N}$. So $\mathscr{B}_{i}$ is lower semicontinuous in $x$. Moreover, if (i) and (ii) are 
satisfied for every $x \in X$, then $\mathscr{B}_{i}$ is lower semicontinuous in $X$.

Step $2\left(\mathscr{B}_{i}\right.$ is upper semicontinuous with closed images). Let $\left(x_{j, v}\right)_{v \in \mathbb{N}}$ be a sequence converging to $x_{j}$ and let $\left(y_{j, v}\right)_{v \in \mathbb{N}}$ be a sequence converging to $y_{j}$, with $y_{j, v} \in K_{i j}\left(x_{j, v}\right)$ for every $v \in$ $\mathbb{N}$. We show that $y_{j} \in K_{i j}\left(x_{j}\right)$. In fact, from $C y_{j, v}^{T} \geqq b^{T}\left(x_{j, v}\right)$ and $\mathbf{1}_{k_{j}} y_{j, v}^{T}=1$ and the continuity of $b(\cdot)$ we get $C y_{j}^{T} \geqq b^{T}\left(x_{j}\right)$ and $\mathbf{1}_{k_{j}} y_{j}^{T}=1$ by taking the limit as $v \rightarrow \infty$. So, $y_{j} \in K_{i j}\left(x_{j}\right)$ and $K_{i j}$ is closed in $x_{j}$. Moreover, if (i) and (ii) are satisfied for every $x_{j} \in X_{j}$, then $K_{i j}$ is closed in $X_{j}$.

Now, let $\left(x_{\nu}\right)_{v \in \mathbb{N}}$ be a sequence converging to $x$ and let $\left(\varrho_{\nu}\right)_{\nu \in \mathbb{N}}$ be a sequence converging to $\varrho$, with $\varrho_{\nu} \in \mathscr{B}_{i}\left(x_{\nu}\right)$ for every $\nu \in \mathbb{N}$. By definition $(21), \varrho_{\nu}(\psi)=x_{i, \nu}\left(\psi_{i}\right)\left[\prod_{j \neq i} y_{j, \nu}\left(\psi_{j}\right)\right]$ for all $\psi \in \Psi$, where $y_{j, \nu} \in K_{i j}\left(x_{j, v}\right)$ for all $j \neq i$. Since $y_{j, v} \rightarrow$ $y_{j}$ and $K_{i j}$ is closed, then $y_{j} \in K_{i j}\left(x_{j}\right)$ for every $j \neq i$. So $\varrho_{\nu} \rightarrow$ $\varrho$, where $\varrho(\psi)=x_{i}\left(\psi_{i}\right)\left[\prod_{j \neq i} y_{j}\left(\psi_{j}\right)\right]$ for all $\psi \in \Psi$. Therefore, (21) implies that $\varrho \in \mathscr{B}_{i}(x)$ and $\mathscr{B}_{i}$ is closed (hence upper semicontinuous) in $x$. Moreover, if (i) and (ii) are satisfied for every $x \in X$, then $\mathscr{B}_{i}$ is closed (hence upper semicontinuous) in $X$. Finally, if $\left(x_{\nu}\right)_{v \in \mathbb{N}}$ is the constant sequence, with $x_{\nu}=x$ for every $v \in \mathbb{N}$, then the closedness of $\mathscr{B}_{i}$ in $x$ implies that the set $\mathscr{B}_{i}(x)$ is closed.

Step $3\left(\mathscr{B}_{i}\left(\cdot, x_{-i}\right)\right.$ is a convex set-valued map, for every $x_{-i} \epsilon$ $\left.X_{-i}\right)$. By definition,

$$
\begin{gathered}
\varrho \in \mathscr{B}_{i}\left(t x_{i}^{\prime}+(1-t) x_{i}^{\prime \prime}, x_{-i}\right) \\
\Longleftrightarrow \exists\left(y_{j}\right)_{j \neq i} \in \prod_{j \neq i} K_{i j}\left(x_{j}\right)
\end{gathered}
$$

s.t. $\varrho(\psi)$

$$
=\left(t x_{i}^{\prime}\left(\psi_{i}\right)+(1-t) x_{i}^{\prime \prime}\left(\psi_{i}\right)\right)\left[\prod_{j \neq i} y_{j}\left(\psi_{j}\right)\right]
$$

$\forall \psi \in \Psi$.

It clearly follows that

$$
\begin{aligned}
\varrho(\psi)= & t x_{i}^{\prime}\left(\psi_{i}\right)\left[\prod_{j \neq i} y_{j}\left(\psi_{j}\right)\right] \\
& +(1-t) x_{i}^{\prime \prime}\left(\psi_{i}\right)\left[\prod_{j \neq i} y_{j}\left(\psi_{j}\right)\right] \quad \forall \psi \in \Psi .
\end{aligned}
$$

Let $\varrho^{\prime}$ and $\varrho^{\prime \prime}$ be defined by

$$
\begin{aligned}
& \varrho^{\prime}(\psi)=x_{i}^{\prime}\left(\psi_{i}\right)\left[\prod_{j \neq i} y_{j}\left(\psi_{j}\right)\right], \\
& \varrho^{\prime \prime}(\psi)=x_{i}^{\prime \prime}\left(\psi_{i}\right)\left[\prod_{j \neq i} y_{j}\left(\psi_{j}\right)\right]
\end{aligned}
$$

Then $\varrho=t \varrho^{\prime}+(1-t) \varrho^{\prime \prime}, \varrho^{\prime} \in \mathscr{B}_{i}\left(x_{i}^{\prime}, x_{-i}\right)$, and $\varrho^{\prime \prime} \in \mathscr{B}_{i}\left(x_{i}^{\prime \prime}\right.$, $\left.x_{-i}\right)$. Since $\varrho$ is arbitrary, then

$$
\begin{aligned}
& \mathscr{B}_{i}\left(t x_{i}^{\prime}+(1-t) x_{i}^{\prime \prime}, x_{-i}\right) \\
& \quad \subseteq t \mathscr{B}_{i}\left(x_{i}^{\prime}, x_{-i}\right)+(1-t) \mathscr{B}_{i}\left(x_{i}^{\prime \prime}, x_{-i}\right) .
\end{aligned}
$$

It follows easily that the previous inclusion holds for every $t \in[0,1]$ and every $x_{i}^{\prime}$ and $x_{i}^{\prime \prime}$ in $X_{i}$. Hence, $\mathscr{B}_{i}\left(\cdot, x_{-i}\right)$ is a convex set-valued map in $X_{i}$, for every $x_{-i} \in X_{-i}$.

The previous proposition shows that the convexity property of the correspondence $\mathscr{B}_{i}$ follows from the definition while the continuity of $\mathscr{B}_{i}$ follows from the continuity of the functions $P_{i j}$ and from a Slater-like condition ${ }^{9}$ : the system of linear inequalities which defines the beliefs of each player has interior points belonging to the simplex (assumption (ii) in the statement of Proposition 7). In order to better understand how much restrictive this latter condition is in our framework, we give an illustrative example which is built upon the example given in Section 2. It is shown in the example below that (ii) is always satisfied except for one point. However, we prove that in this point the correspondence $\mathscr{B}_{i}$ is continuous as well; then, it follows that (ii) is not a necessary condition for the continuity of $\mathscr{B}_{i}$.

Finally, the example below shows the relevance of Proposition 7 in possible applications as it turns out that checking whether (ii) holds is much simpler than checking directly the continuity of $\mathscr{B}_{i}$.

Example 8. Consider the case of a 2-player game constructed as in the example given in Section 2, in which agent $i$ can only perceive the minimum probability under which his opponent $j$ will play each pure strategy; that is

$$
\begin{aligned}
& K_{i j}\left(x_{j}\right)=\left\{y_{j} \in X_{j} \mid y_{j}\left(\psi_{j}^{d}\right) \geqslant \min _{d \in\left\{1, \ldots, k_{j}\right\}} x_{j}\left(\psi_{j}^{d}\right) \forall d\right. \\
& \left.\quad \in\left\{1, \ldots, k_{j}\right\}\right\} \quad \forall x_{j} \in X_{j} .
\end{aligned}
$$

We recall that $K_{i j}\left(x_{j}\right)$ comes out from coherent lower expectations by setting, in formula (2),

$$
\mathscr{Y}_{i j}=\left\{\chi\left(\psi_{j}^{d}\right) \mid d=1, \ldots, k_{j}\right\},
$$

where each $\chi\left(\psi_{j}^{d}\right)$ is the characteristic function of the event $\left\{\psi_{j}^{d}\right\}$ and

$$
P_{i j}\left(\chi\left(\psi_{j}^{d}\right), x_{j}\right)=\min _{d \in\left\{1, \ldots, k_{j}\right\}} x_{j}\left(\psi_{j}^{d}\right) .
$$

Firstly recall that the minimum of a finite number of continuous functions is a continuous function; then it follows that $P_{i j}\left(\chi\left(\psi_{j}^{d}\right), \cdot\right)$ is continuous in the entire set $X_{j}$.

Now, given a point $x_{j} \in X_{j}$, denote with $\mu=$ $\min _{d \in\left\{1, \ldots, k_{j}\right\}} x_{j}\left(\psi_{j}^{d}\right)$. There are two possible cases, either $\mu<$ $1 / k_{j}$ or $\mu=1 / k_{j}$. The case $\mu>1 / k_{j}$ is not possible because 
it would imply that $\sum_{d=1}^{k_{j}} x_{j}\left(\psi_{j}^{d}\right)>1$ which is a contradiction since $x_{j}$ is a probability distribution over the pure strategies of player $j$.

Note that $\mu=1 / k_{j}$ implies that $x_{j}\left(\psi_{j}^{d}\right) \geqslant 1 / k_{j}$ for every $d=1, \ldots, k_{j}$. If there exists $l \in\left\{1, \ldots, k_{j}\right\}$ such that $x_{j}\left(\psi_{j}^{l}\right)>$ $1 / k_{j}$, then $\sum_{d=1}^{k_{j}} x_{j}\left(\psi_{j}^{d}\right)>\sum_{d=1}^{k_{j}}\left(1 / k_{j}\right)=1$, but this is a contradiction since $x_{j}$ is a probability distribution. So, it must be that $x_{j}\left(\psi_{j}^{1}\right)=\cdots=x_{j}\left(\psi_{j}^{k_{j}}\right)=\mu$. Hence, $\mu=1 / k_{j}$ if and only if $x_{j}\left(\psi_{j}^{1}\right)=\cdots=x_{j}\left(\psi_{j}^{k_{j}}\right)=\mu$. Hereafter, we denote with $\bar{x}_{j}$ the strategy in $X_{j}$ defined by $\bar{x}_{j}\left(\psi_{j}^{1}\right)=\cdots=\bar{x}_{j}\left(\psi_{j}^{k_{j}}\right)=\mu$.

Suppose first that $\mu<1 / k_{j}$; let $\varepsilon=\left(1-k_{j} \mu\right) / k_{j}$, and define $y_{j}\left(\psi_{j}^{d}\right)=\mu+\varepsilon$ for every $d \in\left\{1, \ldots, k_{j}\right\}$. Since $\varepsilon>0$, then $y_{j}\left(\psi_{j}^{d}\right)>0$ for each $d$; moreover, it immediately follows that $\sum_{d=1}^{k_{j}} y_{j}\left(\psi_{j}^{d}\right)=1$. Therefore $y_{j}=\left(y_{j}\left(\psi_{j}^{1}\right), \ldots, y_{j}\left(\psi_{j}^{k_{j}}\right)\right) \in X_{j}$. By construction,

$$
\begin{aligned}
& E_{y_{j}}\left[\chi\left(\psi_{j}^{d}\right)\right]=y_{j}\left(\psi_{j}^{d}\right)>\mu=P_{i j}\left(\chi\left(\psi_{j}^{d}\right), x_{j}\right) \\
& \forall d \in\left\{1, \ldots, k_{j}\right\} .
\end{aligned}
$$

It follows that condition (ii) in the assumption of Proposition 7 is satisfied for every point $x_{j} \in X_{j}$ such that $\mu<1 / k_{j}$. That is, the corresponding set-valued map $\mathscr{B}_{i}$ is continuous in every point $\left(x_{i}, x_{j}\right) \in X_{i} \times X_{j}$ such that $x_{j} \neq \bar{x}_{j}$.

Suppose now that $\mu=1 / k_{j}$; that is $x_{j}=\bar{x}_{j}$. Let $y_{j} \in$ $K_{i j}\left(\bar{x}_{j}\right)$; then $y_{j}\left(\psi_{j}^{d}\right) \geqslant 1 / k_{j}$ for every $d \in\left\{1, \ldots, k_{j}\right\}$. If there exists $l \in\left\{1, \ldots, k_{j}\right\}$ such that $y_{j}\left(\psi_{j}^{l}\right)>1 / k_{j}$, then $\sum_{d=1}^{k_{j}} y_{j}\left(\psi_{j}^{d}\right)>1$, but this is a contradiction since $y_{j} \in X_{j}$. Hence $K_{i j}\left(\bar{x}_{j}\right)=\left\{\bar{x}_{j}\right\}$, which immediately implies that assumption (ii) in Proposition 7 is not satisfied as $\bar{x}_{j}\left(\psi_{j}^{d}\right)=P_{i j}\left(\chi\left(\psi_{j}^{d}\right)\right.$, $\left.\bar{x}_{j}\right)$ for all $d \in\left\{1, \ldots, k_{j}\right\}$. Nevertheless, we prove that $K_{i j}$ is continuous in $\bar{x}_{j}$. Let $\left(x_{j, v}\right)_{v \in \mathbb{N}}$ be a sequence converging to $\bar{x}_{j}$. By definition, it follows that $x_{j, v} \in K_{i j}\left(x_{j, v}\right)$ for every $\nu$ and hence $K_{i j}$ is lower semicontinuous in $\bar{x}_{j}$ as $x_{j, v} \rightarrow \bar{x}_{j}$. On the other hand, let $\left(x_{j, v}\right)_{v \in \mathbb{N}}$ be a sequence converging to $\bar{x}_{j}$ and $\left(y_{j, v}\right)_{v \in \mathbb{N}}$ a sequence converging to $y_{j}$ such that $y_{j, v} \in K_{i j}\left(x_{j, v}\right)$ for every $\nu$. Since $X_{j}$ is compact, then $y_{j} \in X_{j}$. Moreover, $y_{j, v} \in K_{i j}\left(x_{j, v}\right)$ implies that

$$
\begin{aligned}
& E_{y_{j, \nu}}\left[\chi\left(\psi_{j}^{d}\right)\right]=y_{j, \nu}\left(\psi_{j}^{d}\right) \geqslant P_{i j}\left(\chi\left(\psi_{j}^{d}\right), x_{j, \nu}\right) \\
& \forall d \in\left\{1, \ldots, k_{j}\right\} .
\end{aligned}
$$

Taking the limit for $v \rightarrow \infty$, it follows from the continuity of the functions $P_{i j}$ that

$$
\begin{aligned}
E_{y_{j}}\left[\chi\left(\psi_{j}^{d}\right)\right] & =\lim _{\nu \rightarrow \infty} E_{y_{j, \nu}}\left[\chi\left(\psi_{j}^{d}\right)\right] \\
& \geqslant \lim _{\nu \rightarrow \infty} P_{i j}\left(\chi\left(\psi_{j}^{d}\right), x_{j, v}\right) \\
& =P_{i j}\left(\chi\left(\psi_{j}^{d}\right), \bar{x}_{j}\right) \quad \forall d \in\left\{1, \ldots, k_{j}\right\},
\end{aligned}
$$

which implies that $y_{j} \in K_{i j}\left(\bar{x}_{j}\right)$ (in particular $\left.y_{j}=\bar{x}_{j}\right)$. Therefore, $K_{i j}$ is a closed and hence continuous set-valued map in $\bar{x}_{j}$. Following the same steps of the proof of Proposition 7 , we can prove that if $K_{i j}$ is continuous in $\bar{x}_{j}$ then $\mathscr{B}_{i}$ is continuous in $\left(x_{i}, \bar{x}_{j}\right)$ for every $x_{i} \in X_{i}$. As a final remark, the example emphasizes that it is more simple to check our Slater-like condition rather than applying the definition of upper and lower semicontinuity for correspondences, even in this particular case in which there is a unique point $\bar{x}_{j}$ in which the Slater-like condition is not satisfied and the setvalued map $K_{i j}$ in this point is single valued. It is clear that, in more complex cases, calculations would have been even more demanding.

Remark 9. In order to keep the presentation of Proposition 7 more simple, we did not consider the case in which there exist implicit linear equalities in the system of linear inequalities. However, the generalization to this case is rather straightforward. It consists in adding the assumption that the coefficient matrix of the system of the implicit equalities together with the equation in (23) has full rank. The proof is substantially identical to the one given above.

\section{Conflict of Interests}

The authors declare that there is no conflict of interests regarding the publication of this paper.

\section{Acknowledgments}

This research was carried out in the frame of Programma STAR Napoli Call 201389 "Equilibrium with ambiguity," financially supported by UniNA and Compagnia di San Paolo. Moreover, the authors acknowledge the financial support of PRIN 20103S5RN3 "Robust decision making in markets and organizations."

\section{Endnotes}

1. Indeed, in Walley [12] this statement is supported by illustrative examples.

2. In De Marco and Romaniello [15] the equivalent formulation of equilibria with partially specified probabilities as equilibria in games under ambiguous beliefs correspondences is given.

3. De Marco and Romaniello [11] present results of existence and stability for the equilibria in games under ambiguous beliefs correspondences under relaxed assumptions on players' preferences. These results could be useful to study generalizations of the analysis presented in this paper. However, here we focus on a minor departure from Lehrer's analysis in order to better highlight the differences between the two approaches.

4. All the definitions and the propositions we use, together with the proofs, can be found in this book. 
5. Every set-valued map in this paper satisfies the assumptions of this result. Hence, upper semicontinuity and closedness coincide in this work.

6. Note that a set-valued map is concave if and only if its graph is a convex set. For this reason, some authors call convex set-valued maps those that here we call concave.

7. Indeed, this is more clearly explained in Daniel [16] (Section 4, page 771).

8. It is clear from Robinson's [14] definition of stability at page 755 that $\delta$ is uniform (it does not depend on $y_{j}$ ) because the perturbation does not involve the matrix $C$.

9. The Slater condition plays an important role in nonlinear programming, being one of the possible constraints qualification assumptions in the first order necessary conditions theorems for local optima.

\section{References}

[1] J. W. Friedman and C. Mezzetti, "Random belief equilibrium in normal form games," Games and Economic Behavior, vol. 51, no. 2, pp. 296-323, 2005.

[2] J. Dow and S. R. Werlang, "Nash equilibrium under Knightian uncertainty: breaking down backward induction," Journal of Economic Theory, vol. 64, no. 2, pp. 305-324, 1994.

[3] J. Eichberger and D. Kelsey, "Non-additive beliefs and strategic equilibria," Games and Economic Behavior, vol. 30, no. 2, pp. 183-215, 2000.

[4] M. Marinacci, "Ambiguous games," Games and Economic Behavior, vol. 31, no. 2, pp. 191-219, 2000.

[5] J. Eichberger, D. Kelsey, and B. C. Schipper, "Ambiguity and social interaction," Oxford Economic Papers, vol. 61, no. 2, pp. 355-379, 2009.

[6] K. C. Lo, "Equilibrium in beliefs under uncertainty," Journal of Economic Theory, vol. 71, no. 2, pp. 443-484, 1996.

[7] P. Klibanoff, "Uncertainty, decision, and normal form games," Working Paper, 1996.

[8] E. Groes, H. J. Jacobsen, B. Sloth, and T. Tranaes, "Nash equilibrium with lower probabilities," Theory and Decision, vol. 44, no. 1, pp. 37-66, 1998.

[9] E. Lehrer, "Partially specified probabilities: decisions and games," American Economic Journal: Microeconomics, vol. 4, no. 1, pp. 70-100, 2012.

[10] G. De Marco and M. Romaniello, "Beliefs correspondences and equilibria in ambiguous games," International Journal of Intelligent Systems, vol. 27, no. 2, pp. 86-107, 2012.

[11] G. De Marco and M. Romaniello, "Variational preferences and equilibria in games under ambiguous beliefs correspondences," International Journal of Approximate Reasoning, vol. 60, pp. 822, 2015.

[12] P. Walley, "Towards a unified theory of imprecise probability," International Journal of Approximate Reasoning, vol. 24, no. 2-3, pp. 125-148, 2000.

[13] J. P. Aubin and H. Frankowska, Set Valued Analysis, Birkhäuser, Boston, Mass, USA, 1990.

[14] S. M. Robinson, "Stability theory for systems of inequalities. Part I. Linear systems," SIAM Journal on Numerical Analysis, vol. 12, no. 5, pp. 754-769, 1975.
[15] G. de Marco and M. Romaniello, "A limit theorem for equilibria under ambiguous belief correspondences," Mathematical Social Sciences, vol. 66, no. 3, pp. 431-438, 2013.

[16] J. W. Daniel, "Remarks on perturbations in linear inequalities," SIAM Journal on Numerical Analysis, vol. 12, no. 5, pp. 770-772, 1975. 


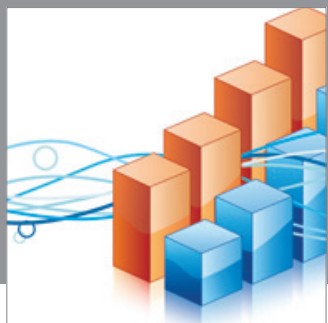

Advances in

Operations Research

mansans

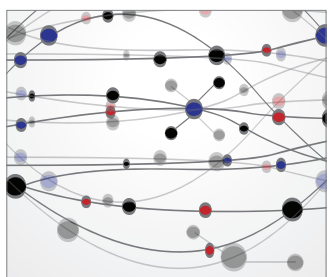

The Scientific World Journal
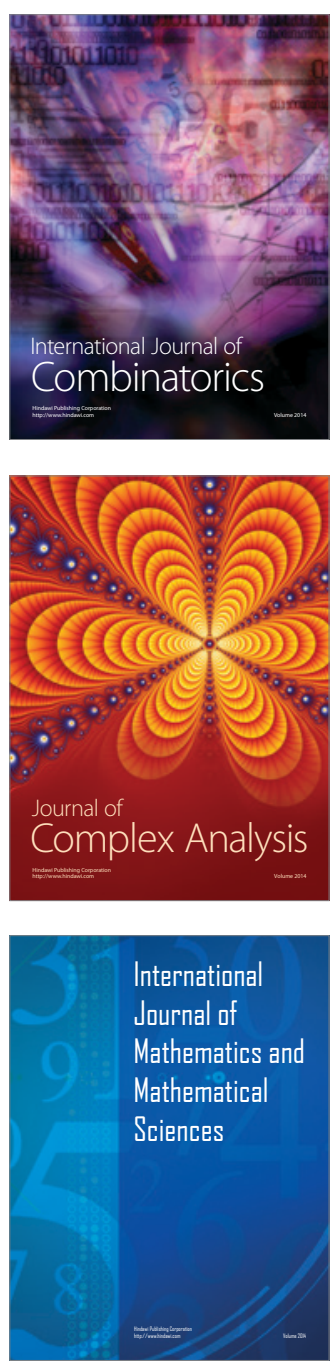
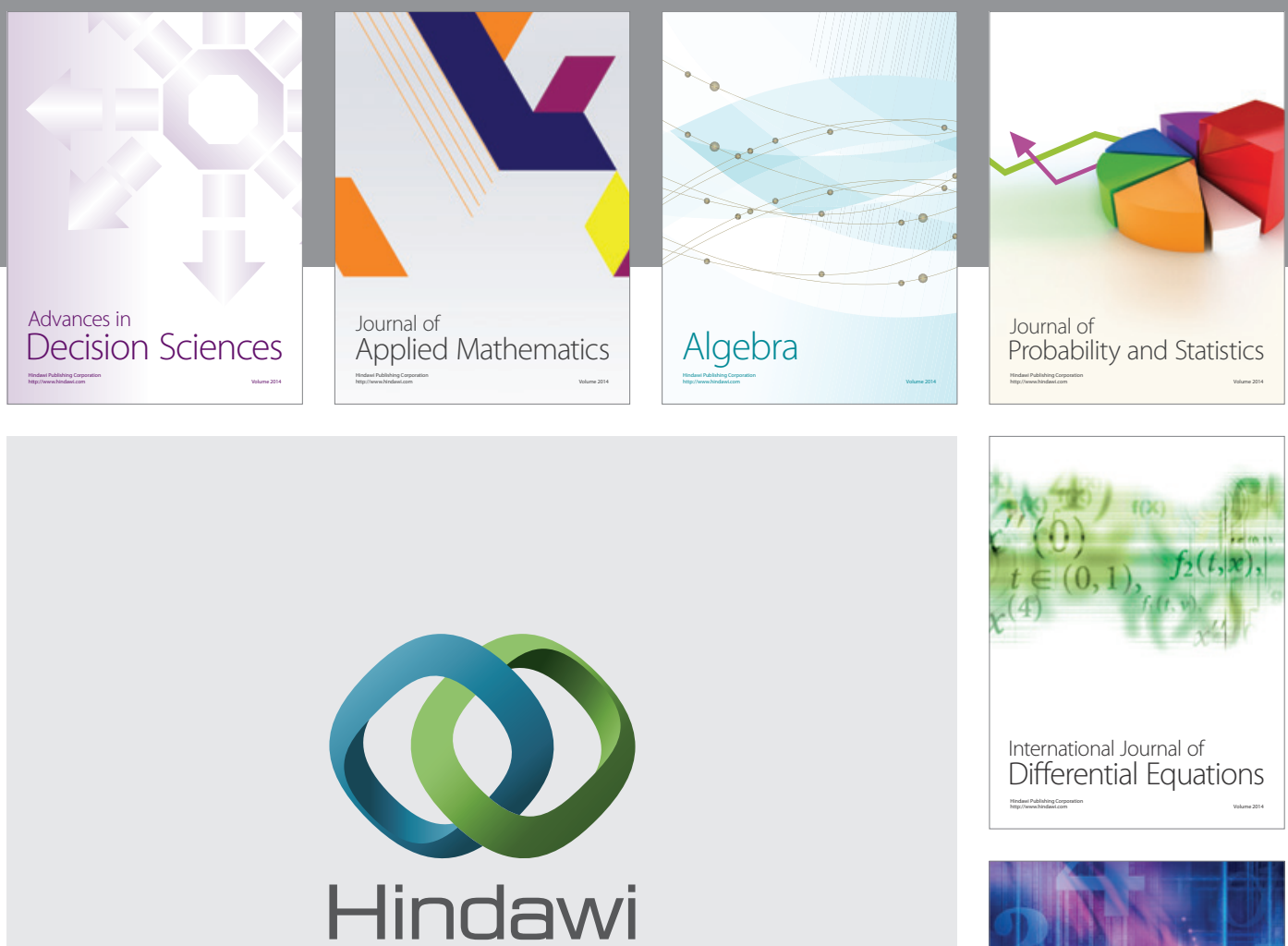

Submit your manuscripts at http://www.hindawi.com
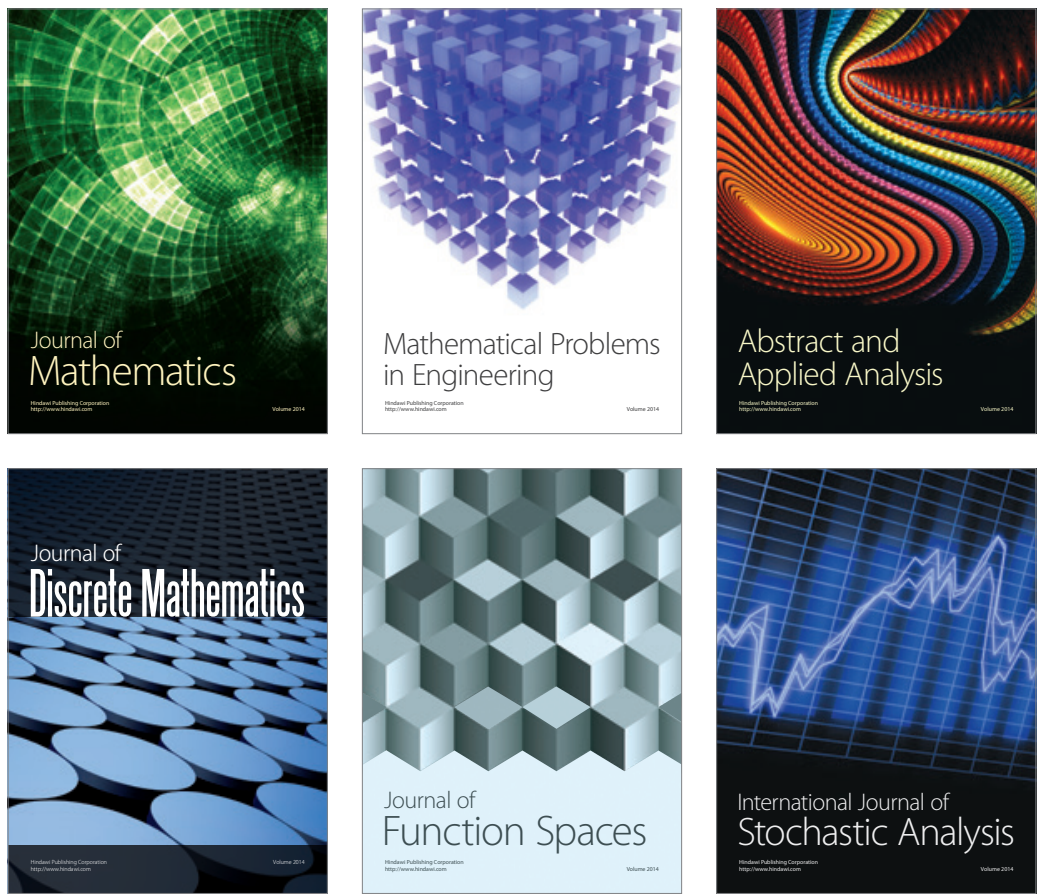

Journal of

Function Spaces

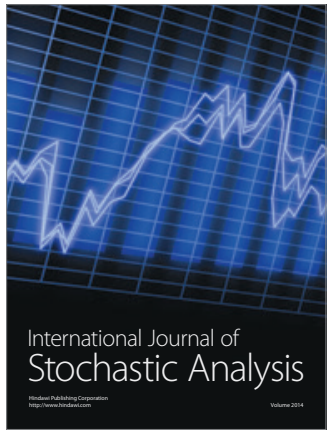

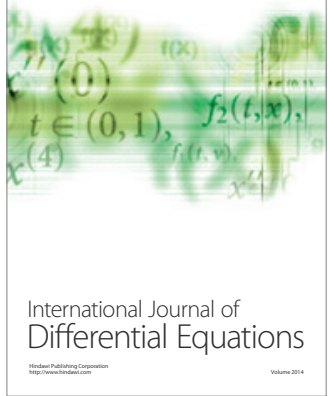
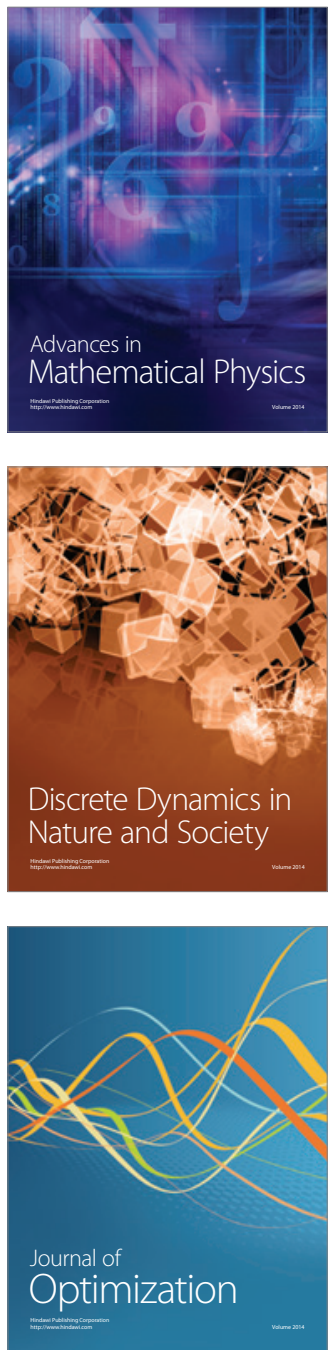